SCIENCE CHINA

Physics, Mechanics \& Astronomy

\title{
Modeling of mechanical behaviors for natural fiber reinforced composites under hygrothermal ageing
}

\author{
Zheng Zhong ${ }^{1,2 *}$, and Fang Tian $^{2}$ \\ ${ }^{1}$ School of Science, Harbin Institute of Technology, Shenzhen 518055, China; \\ ${ }^{2}$ School of Aerospace Engineering and Applied Mechanics, Tongji University, Shanghai 200092, China
}

Received August 24, 2017; accepted September 13, 2017; published online October 27, 2017

$\begin{array}{ll}\text { Citation: } & \text { Z. Zhong, and F. Tian, Modeling of mechanical behaviors for natural fiber reinforced composites under hygrothermal ageing, Sci. China-Phys. Mech. } \\ \text { Astron. 60, } 124631 \text { (2017), doi: 10.1007/s11433-017-9103-3 }\end{array}$

In recent years, natural fiber reinforced composites have been widely applied to various industrial products for their excellent environmental-friendly performance. It is essential to understand the mechanical properties of natural fiber reinforced composites under their in-service environment. Compared with synthetic fibers, the hydrophilicity of natural fibers could result in a much larger quantity of water absorption from the moisture atmosphere, which would have adverse consequences for the durability of natural fiber reinforced composites [1]. The environmental temperature would affect the water absorption process, and furthermore, high temperature may break down the fibrous tissues. Therefore, the effect of hydrothermal ageing on the mechanical behaviors of natural fiber reinforced composites attracts great attention from researchers.

The changes in mechanical behaviors caused by water absorption of various natural fiber reinforced composites, such as composites with fibers of bamboo, hemp, jute or flax, are investigated by means of mechanical measurements and scanning electron microscopy [2]. Experimental studies show that the volumetric swelling of natural fibers accompanies by water absorption process, which would weaken the interface between the natural fibers and the polymer matrix and then leads to the initiation and propagation of cracks. Moreover, besides the physical deterioration caused by the volumetric swelling of natural fibers, the hydrolysis reaction between the natural

*Corresponding author (email: zhongzheng@hit.edu.cn) fibers and the water absorbed plays an important role on the mechanical properties of composites as well. The hydrolysis reaction, occurring for long hygrothermal ageing periods, irreversibly affects the natural fibrous structures and causes debonding between the natural fibers and the polymer matrix.

For experimental observations, some researchers aim at quantitively modeling the evolution of mechanical behaviors of natural fiber reinforced composites during the hygrothermal ageing process to predict their durability in service. On the basis of the non-equilibrium thermodynamic framework, Pan and Zhong [3-7] have conducted a series of research on modeling the effect of moisture absorption on the mechanical properties of natural fiber reinforced composites. In their analyses, the internal variables are introduced into the Helmholtz free energy to describe the mechanical degradation during the water absorption stage in the hygrothermal ageing process, and the relation between the amount of the water absorbed by the composites and the volumetric swelling of the composites is formulated on the hypothesis of the elastic incompressibility. For example, for the unidirectional natural fiber reinforced composites considering moisture absorption, the specific Helmholtz free energy function is given by

$$
\begin{aligned}
\psi= & \frac{1}{2} \boldsymbol{G}\left(1-\beta_{1} \alpha\right)\left(\bar{I}_{1}-3\right)+\frac{1}{2} c \boldsymbol{G} \gamma\left(1-\beta_{2} \alpha\right)\left(\bar{I}_{4}-1\right)^{2} \\
& -c \boldsymbol{G} \gamma \ln J
\end{aligned}
$$

where $G$ is the shear modulus of the matrix and $\gamma$ is the relative shear modulus of the natural fiber to the matrix, $c$ is the nat- 
ural fiber content in the composites, $\beta_{1}$ and $\beta_{2}$ represent the interface degradation parameter and the fiber degradation parameter, respectively. $\alpha$ denotes the damage variables. $\bar{I}_{1}$ and $\bar{I}_{4}$ are the principal invariant of modified right Cauchy-Green tensor and the pseudo-invariants of modified Cauchy-Green tensor, respectively. $J$ is the volume ratio. They established the damage models for the evolutions of the mechanical properties of natural fiber reinforced composites. These damage models take into account the nonlinear constitutive relations of the materials, the large deformation behaviors caused by moisture absorption and the different distributions of natural fibers in the composites with unidirectional natural fibers, short natural fibers and hybrid natural short fibers. Their theoretical predictions are in good agreement with corresponding experimental results (Figure 1), which shows that the elastic modulus decrease rapidly with water absorption at initial stage and then slow down gradually when the molar concentration of water molecules approaches to saturation in the composites.

Moreover, in order to reveal the moisture absorption related damage mechanisms from the microscopic view, Pan and Zhong [8] applied the micromechanical approach to model the mechanical performance of natural fiber reinforced composites with volumetric swelling induced by moisture absorption. They modified the Mori-Tanaka method by introducing a damage variable and established a set of micromechanical equations to describe the mechanical degradation of the composites during the water absorption process. It is proved by the experimental observations that the micromechanical model is capable of predicting the modulus loss with the increasing water concentration. Pan and Zhong [3-7] have built a series of theoretical models to analyze the mechanical behaviors of natural fiber reinforced composites during the moisture absorption process from both macro and micro aspects.

Since the hydrolysis reaction between the natural fibers and

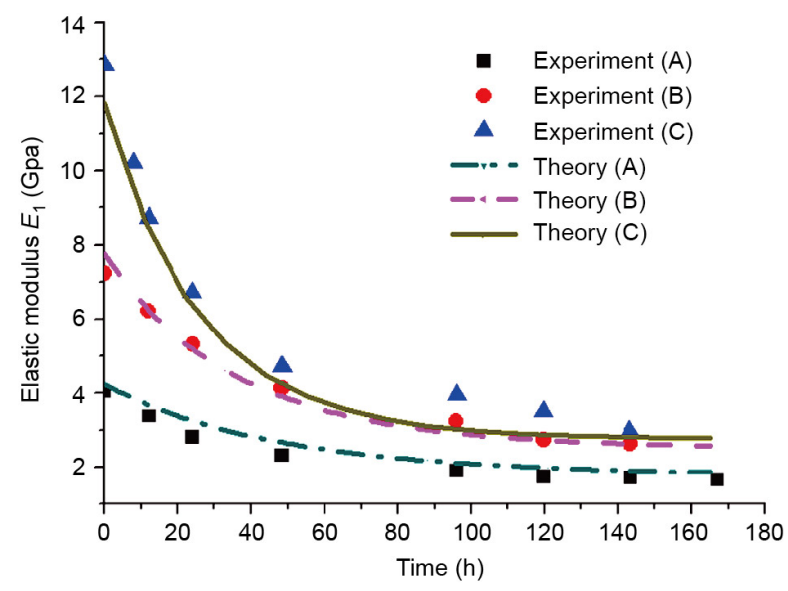

Figure 1 (Color online) Evolution of elastic modulus of sisal fiber reinforced composites with three different fiber contents (A: 10.2\%; B: 19.7\%; C: $30.4 \%)[7]$. the water molecules also plays an important role for long ageing times, Tian et al. [9] gave a modeling on the mechanical behaviors of natural fiber reinforced composites when considering the influence of both the moisture absorption and the hydrolysis reaction. Two internal variables are introduced into the nonlinear constitutive model to represent the damages caused by the volumetric swelling during the water absorption process and the hydrolysis reaction during the long-term ageing, respectively, that is

$\psi=\widetilde{\psi}\left(\bar{C}, J, \alpha_{s}, \alpha_{l}\right)$,

where $\bar{C}$ is the modified Cauchy-Green tensor, $\alpha_{s}$ and $\alpha_{l}$ represent the damage variable related to water absorption and hydrolysis reaction, respectively. The evolution laws for these two internal variables are formulated. This theoretical model is in accord with experimental observations (Figure 2), which shows that the water absorption may reach its saturation in the composites for just a few weeks but the modulus loss may last for two or three years or even longer. The hydrolysis reaction is at work in the mechanical properties of natural fiber reinforced composites, especially for long ageing times in hygrothermal environment.

Understanding the mechanical behaviors of natural fiber reinforced composites under hygrothermal ageing is vital for their applications, especially for those services in humid environment. Modeling their constitutive relations is the basis of prediction for their durability in service. Till now, we have established several theoretical constitutive models to analyze and predict the mechanical behaviors of natural fiber reinforced composites under hygrothermal ageing, which are proved to be useful and practical by compared with corresponding experimental results.

Yet, there is still scope for researchers to break through to further improve and perfect the theoretical models of natural fiber reinforced composites under long ageing times. For ageing of natural fiber reinforced composites is a physical and

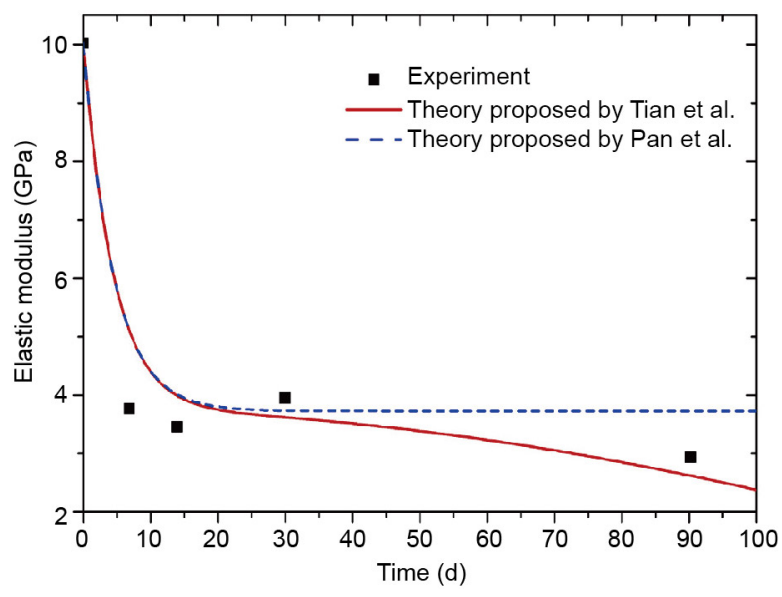

Figure 2 (Color online) Evolution of elastic modulus of ramie fiber reinforced composites [9]. 
chemical process rather than an exclusively physical process [10], it is desirable to introduce the thermo-chemo-elastic coupled theory proposed by Zhang and Zhong [11,12] into the future modeling studies of natural fiber reinforced composite. The general Helmholtz free energy could be expressed in the following form

$\psi=\widetilde{\psi}\left(\varepsilon, \varphi_{a}, T, \ell_{\gamma}\right)$.

In this form, the extent of chemical reaction $\ell_{\gamma}$ is introduced as an independent state variable. Macroscopic mechanical testing for natural fiber reinforced composites under long-term hygrothermal ageing times and experimental researches on change in microstructure of natural fibers during the ageing process are also important for future studies. Moreover, how to analyze and predict the durability of components made by natural fiber reinforced composites in complicate environmental and loading conditions by the finite element method would be a hot issue as well, which are considered to be multiphysics coupling problems.

As the mechanical performance of composites depends to a great extent on the fiber-matrix interface, the interface treatment of the composite materials should be taken into consideration in developing the hygrothermal ageing constitutive models by introducing interface models, such as the springtype interface model employed in Shi et al. [13], or formulating some appropriate interface models that are capable of describing the effect of the interface adhesions and defects.

Furthermore, the present theoretical models are mainly focused on the elastic responses of natural fiber reinforced composites under hygrothermal ageing for it is hard to predict the strength of materials by the continuum mechanics approach. Nevertheless, the strength of materials is also an important criterion to evaluate the materials' durability. The molecular dynamics methods developed rapidly in recent years make it possible for researchers to understand the plastic deformation and fracture process of fiber reinforced composites [14]. Anyway, how to improve strength of natural fiber reinforced composites without loss of ductility is worthy of further research likewise [15].

Up till now, it has made some achievements on modeling the mechanical behaviors of natural fiber reinforced composites under hygrothermal ageing, which yet deserves more researches than it has received.

This work was supported by the Shenzhen Municipal Government through the Fundamental Research Project (Grant No. JCYJ20170307151049286) and the National Natural Science Foundation of China (Grant No. 11572227).

1 D. Scida, M. Assarar, C. Poilâne, and R. Ayad, Compos. Part B-Eng. 48, 51 (2013).

2 M. Assarar, D. Scida, A. El Mahi, C. Poilâne, and R. Ayad, Mater. Des. 32, 788 (2011).

3 Y. H. Pan, and Z. Zhong, Sci. China-Phys. Mech. Astron. 59, 664603 (2016).

4 Y. Pan, and Z. Zhong, J. Mech. Mater. Struct. 10, 79 (2015).

5 Y. Pan, and Z. Zhong, Compos. Sci. Tech. 110, 132 (2015).

6 Y. Pan, and Z. Zhong, Compos. Sci. Tech. 103, 22 (2014).

7 Y. Pan, and Z. Zhong, J. Mech. Phys. Solids 69, 132 (2014).

8 Y. Pan, and Z. Zhong, Mech. Mater. 85, 7 (2015).

9 F. Tian, Y. Pan, and Z. Zhong, Compos. Sci. Tech. 142, 156 (2017).

10 M. P. Foulc, A. Bergeret, L. Ferry, P. Ienny, and A. Crespy, Polym. Degrad. Stab. 89, 461 (2005)

11 X. L. Zhang, and Z. Zhong, Sci. China-Phys. Mech. Astron. 60, 084611 (2017).

12 X. Zhang, and Z. Zhong, J. Mech. Phys. Solids 107, 49 (2017).

13 Y. Shi, Y. P. Wan, and Z. Zhong, Sci. China-Phys. Mech. Astron. 59, 104611 (2016)

14 Z. Y. Yang, J. Sun, Z. X. Lu, and W. J. Hu, Sci. China-Phys. Mech. Astron. 59, 634603 (2016).

15 C. Y. Liu, M. Z. Ma, R. P. Liu, L. Yu, and K. Luo, Sci. China-Phys. Mech. Astron. 58, 104601 (2015). 